\title{
CUIDADOS EN LOS PRIMEROS MIL DÍAS DE VIDA. ARMENTA, SAN PEDRO SULA.
}

\author{
Care in the first thousand days of life, Armenta. San Pedro Sula.
}

*Vilma Mercedes Miranda Baquedano, ${ }^{* *}$ Lucia Alejandra Canales Bonilla, ${ }^{* *}$ Ena Melissa Padilla Benegas.

\section{RESUMEN}

Los mil días de vida van desde la concepcion hasta los 2 años de edad, etapa donde se determina el desarrollo básico del niño; la falta de una alimentación y cuidados adecuados produce daños físicos y cognitivos irreversibles para el resto de su vida. Objetivo Identificar los cuidados ofrecidos por la madre/encargado en los primeros mil días de vida, en los niños del programa de Atencion Integral de la niñez en la comunidad (AIN-C) de Armenta, San Pedro Sula, I Semestre académico del 2016. Pacientes y Método: Investigación cuantitativa, transversal, descriptivo, instrumento tipo cuestionario aplicado a madres/encargados del cuidado de niños menores de dos años monitoreados por el programa AIN-C, estrategia de la secretaria de salud (SESAL) Población: 69 madres/encargados, muestra: 60 que accedieron a participar. Resultados: $83.4 \%$ de los niños menores de 2 años son cuidados por la madre, $83.3 \%$ asistieron a control prenatal, $66.6 \%$ de las madres asistió a un control puerperal, $93.4 \%$ de los menores recibieron lactancia materna/mixta, $100 \%$ de las madres/encargados vacunaron a los menores y $56.6 \%$ integraron los alimentos antes de los 6 meses. Conclusiones Los resultados señalan que las madres/encargados no realizan todos los cuidados que se deben ofrecer al niño en los primeros mil días de vida y los que se ofrecen no reúnen los requerimientos necesarios que le garanticen un desarrollo físico y cognitivo adecuado que le permita estar saludable en las siguientes etapas de su vida.

*Profesora de la Carrera de Enfermería de la Escuela Universitaria de Ciencias de la salud (EUCS) de la Universidad Nacional Autónoma de Honduras en el Valle de Sula (UNAH-VS). Licenciada en Enfermería. Especialista en Salud Materno Perinatal. Magister en Gestión Educativa.

** Estudiantes de la Carrera de Enfermería de la EUCS-UNAHVS.

Dirigir correspondencia a: vilmamiranda2012@yahoo.es

Recibido: 10 de agosto 2017

Aprobado: 26 de octubre 2017

\section{PALABRAS CLAVE}

Cuidadores, estrategias, niño.

\section{ABSTRACT}

The thousand days of life from conception to 2 years of age and basic development of the child is determined, lack of adequate food and care causes irreversible physical and cognitive damage the child for the rest of his life. Objective to identify the care given by the mother / caregiver in the first thousand days of life in children comprehen sive childhood care program Armenta community, San Pedro Sula I half of 2016. Material and Method quantitative, transversal, descriptive research, using an instrument type questionnaire mothers / caregivers of children under two years of community Armenta AIN-C program (Comprehensive care of children in the community) Population: 69 mothers / guardians, sample: 60 agreed to participate. Results $83.4 \%$ of care in children under 2 years it is provided by the mother, $83.3 \%$ attended prenatal care, $66.6 \%$ of mothers attended a puerperal control, $93.4 \%$ of children received breast / MBF, $100 \%$ of the mothers / caregivers attended vaccinate children and $56.6 \%$ integrated food before 6 months. Conclusions The results show that the care provided by the parent / guardian in the first thousand days of life are poor and there is no compliance with the basic and essential beware on children.

\section{KEYWORDS}

Caregivers, strategies, Child.

\section{INTRODUCCIÓN}

Los primeros mil días de vida constituyen el período que sienta las bases para el desarrollo del ser humano, es una etapa que va desde la gestación hasta los dos años de edad, comprende 270 días desde la concep- 
ción hasta el nacimiento, 365 días del primer año de vida y 365 días del segundo año de vida. En este período ocurren numerosos procesos biológicos, psicoafectivos, sociales y culturales que impactan el crecimiento y desarrollo físico, cognitivo, del lenguaje, emocional y social de las niñas y niños, por lo cual se trata de un periodo de alta sensibilidad y vulnerabilidad que exige el cuidado necesario por parte de los adultos. ${ }^{(1)}$

El ambiente alrededor del bebé juega un rol muy importante en los primeros 1000 días de vida, entre los aspectos que más repercuten en el desarrollo y salud futuros de una persona podemos mencionar; cuidados de la mamá durante el embarazo (estado nutricional, control del peso, suplementación vitamínica), lactancia materna exclusiva por 6 meses y prolongada hasta los 2 años, alimentación complementaria adecuada, controles pediátricos (vacunas, estimulación temprana) y cuidados de la salud del bebe, juegos y estimulación al bebé y el cariño de los padres y de quienes lo rodean. ${ }^{(1)}$

Existe evidencia científica acerca de los efectos de las intervenciones tempranas, basadas, entre otras, en la alimentación con leche materna exclusiva en los primeros seis meses de vida y continuada hasta los dos años con alimentos complementarios apropiados; la vacunación para prevenir enfermedades agudas de la infancia y aún enfermedades posteriores para la vida. Las interacciones y el vínculo afectivo con los padres y cuidadores/as garantizan un adecuado crecimiento y el desarrollo de las niñas y niños. En esta etapa tiene lugar el $90 \%$ del proceso de formación y desarrollo del cerebro, que es el órgano rector de las funciones cognitivas, sensoriales, emocionales y motoras que nos permiten explorar el mundo, aprender $y$ transformarlo. ${ }^{(1)}$

Los primeros dos años de la vida del niño son especialmente importantes, puesto que la nutrición óptima durante este período reduce la morbilidad y mortalidad, así como el riesgo de enfermedades crónicas, y mejora el desarrollo general. ${ }^{(2)}$

Honduras, es considerada uno de los países más pobres de América Latina; donde la mitad de la población vive en extrema pobreza y está aquejada por la inseguridad alimentaria (según el Informe sobre Desarrollo Humano 2013 del Programa de las Naciones Unidas para el Desarrollo PNUD). Se ha registrado un alto índice de niños con bajo peso al nacer y tasas elevadas de infecciones respiratorias agudas y diarreas, infecciones que al ser frecuentes y permanecer por largos períodos ocasionan un grave deterioro nutricional, 29 de cada 1,000 niños nacidos en Honduras corren el riesgo de morir antes de los cinco años. ${ }^{(3)}$

Por esta situación es necesario la puesta en marcha de la atención integral a la niñez comunitaria, estrategia que se basa en el reconocimiento del crecimiento y desarrollo de los niños y niñas como un indicador de salud y bienestar. En este contexto, vigilamos la salud de los niños y niñas en los primeros mil días de vida; en donde desarrollamos destrezas de comunicación, negociación y consejería que garanticen cambios en el comportamiento; aplicamos micronutrientes para apoyar el crecimiento adecuado de los niños y niñas e involucramos a las comunidades y gobiernos locales. ${ }^{(4)}$

\section{Cuidados durante la Gestación (primeros 270 días).}

Cuidado prenatal: El control prenatal es un conjunto de acciones médicas y asistenciales que se concretan en entrevistas o visitas programadas con el equipo de salud, a fin de controlar la evolución del embarazo y obtener una adecuada preparación para el parto y la crianza del recién nacido con la finalidad de disminuir los riesgos de este proceso fisioló$\operatorname{gico}^{(5)}$ 
Actividades durante el control prenatal:

- Administración de ácido fólico en el período preconcepcional y en el primer trimestre del embarazo.

- Evaluación de factores de riesgo en cada consulta.

- Determinar el grupo sanguíneo y el factor Rh.

- Evaluar el estado nutricional materno.

- Preparar a la mujer para la maternidad.

- Detectar tabaquismo y otras adiccines.

- Suplementación con hierro.

- Vacunación antitetánica

- Detectar presentación pelviana al término

- Estudio ecográfico

- Papanicolaou y Colposcopía.

- Examen mamario de rutina. ${ }^{(4)}$

- Estimulación intrauterina.

La estimulación intrauterina "se define como dar al futuro bebé excelentes condiciones que le permitan desarrollarse mejor según su proceso natural, su propia dinámica y desarrollar todas las capacidades y facultades que posee en su carga genética, que va a depender del grado de compromiso que tenga tanto la madre como el padre con su bebé. La estimulación prenatal es uno de los cuidados claves en el desarrollo psicomotor del niño: "Mientras aún está en su útero el bebé siente, oye, ve, saborea, responde y hasta aprende y recuerda. ${ }^{(6)}$

\section{Cuidados en el primer año de Vida (sigui- entes 365 días).}

Cuidados del recién nacido: Apego precoz y alojamiento conjunto: Es el inicio de la integración madre-hijo desde los primeros minutos de vida. Favorece el inicio exitoso de la lactancia materna y el vínculo emocional madre-hijo es un derecho universal y recomendado por la OMS. Una buena relación de apego les aporta las armas emocionales adecuadas para una buena adaptación y relación social. ${ }^{(7)}$

El cuidado de la piel del recién nacido: Es especialmente delicado ya que carece de flora bacteriana en el momento del nacimiento $y$ existe en ella una herida fisiológica (cordón umbilical) y en ocasiones dos, en los varones circuncidados por lo que es necesario realizar un aseo frecuente y prolijo del cordón umbilical hasta que éste se caiga y el orificio umbilical cicatrice completamente, la colonización bacteriana umbilical se ha relacionado con onfalitis, sepsis y otras infecciones neonatales, el cuidado del ombligo y piel es necesario para evitar que la colonización alcance a niveles suficiente para desencadenar la infección. ${ }^{(8)}$

La alimentación en el primer año de vida: según la Organización Mundial de la Salud recomienda iniciar la lactancia materna en la primera hora de vida, y mantenerla como única forma de alimentación durante los 6 meses siguientes; a partir de entonces se recomienda seguir con la lactancia materna hasta los 2 años, como mínimo, complementada adecuadamente con otros alimentos inocuos. Si todos los niños de 0 a 23 meses estuvieran amamantados de forma óptima, cada año se les podría salvar la vida a más de 800000 niños de menos de 5 años. La lactancia materna mejora el coeficiente intelectual y la asistencia a la escuela, además de asociarse a mayores ingresos en la vida adulta. ${ }^{(9)}$

Vacunación: La inmunización es una medida urgente que todo niño necesita haber recibido una serie de vacunas antes de cumplir el primer año de vida. Todo recién nacido mayor de 2500 gramos se debe aplicar las dosis de BCG (contra la tuberculosis) y Hepatitis B, a los dos meses de edad se inicia el esquema de vacunación con la primera dosis de polio oral y pentavalente (difteria, tosferina, tétanos, hepatitis B y Haemophilus Influenza tipo b), la primera de neumococo (que protege contra enfermedades como la meningitis y la neumonía) y el rotavirus, a los cuatro meses se aplica las segundas dosis de polio, neumococo pentavalente y rotavirus y a los seis meses la tercera dosis de polio, pentavalente, neumococo y vitamina $A$, a los 12 meses se aplica la primera de SRP (sarampión, rubeola y parotiditis) a los 18 meses se aplican los primeros refuerzos de polio y DPT (difteria, tosferina y tétanos) y 
a los 5 años se aplican los segundos refuerzos de polio y DPT. En la actualidad, la inmunización evita anualmente entre 2 y 3 millones de defunciones y se estima que 18,7 millones de lactantes de todo el mundo aún no reciben las vacunas básicas. ${ }^{(10)}$

\section{Cuidados en el segundo año de vida (siguientes 365 días de vida).}

Alimentación: desde el año de edad el niño/a debe paulatinamente incorporarse a los hábitos y características de la alimentación familiar. Es aconsejable incorporar cuatro tiempos de comida principales durante el día, suspendiendo el horario nocturno de alimentación láctea (desayuno, almuerzo, once y cena), incorporándose a la comida propia del hogar, de consistencia molida y resguardando siempre que ésta sea saludable. En algunos ambientes además el niño recibe un $5^{\circ}$ tiempo de alimentación a media mañana (colación, merienda). ${ }^{(11)}$

En nuestra realidad debiera educarse para retardar al máximo el inicio del consumo de golosinas, evitando en los primeros 2 años de vida; jugos envasados o gaseosas, galletas de cualquier tipo, cereales azucarados, chocolates, confites o helados y productos salados, orientando a que las colaciones o meriendas que llevan los niños pre-escolares cuando asisten a un jardín infantil, sean de preferencia en base a frutas. ${ }^{(11)}$

La importancia de la lactancia materna en el segundo año de vida sigue siendo poco estudiada en comunidades con mejor desarrollo socioeconómico. Seguimos recomendando su continuación mientras el niño mantenga un buen crecimiento, desarrolle hábitos saludables de alimentación y sea gratificante para la madre e hijo. ${ }^{(11)}$

Objetivo Identificar los cuidados brindados por la madre/encargado en los primeros mil días de vida en los niños de la comunidad de Armenta, San Pedro Sula I semestre del 2016.

\section{PACIENTES Y METODOS}

Se realizó un estudio cuantitativo de tipo des- criptivo transversal que pretende Identificar los cuidados brindados por la madre o encargado en los primeros mil días de vida en los niños de la comunidad Armenta, San Pedro Sula. El universo fue de 69 niños menores de dos años de edad que participaron en el programa AINC (atención integral de niñez en la comunidad), la muestra fueron 60 niños/as de madres/encargados que accedieron a participar en la investigación. Se realizó un instrumento tipo cuestionario que contaba con 30 preguntas abiertas y cerradas en las que se evaluó 3 variables:

1. Biopsicosocial del cuidador

2. Cuidados en los primeros mil días del niño 3. Estimulación.

Criterios de inclusión: menores de 2 años que estaban en el programa de AINC.

Criterios de exclusión: niños con más de 2 años, y niños que no estuvieran en el programa de AINC y cuidadores que no quisieran participar.

La aplicación de la encuesta se llevó a cabo con las madres y encargados de los niños menores de dos años en la comunidad Armenta, previo consentimiento informado, los datos fueron analizados a través de los programas Microsoft Excel y Microsoft Word.

\section{RESULTADOS}

El nivel de escolaridad que tenían las madres o encargados era: $41.6 \%$ (25) primaria completa, 38.4\% (23) primaria incompleta, 15\% (9) secundaria incompleta, 3.4\% (2) universidad y $1.6 \%$ (1) termino su secundaria completa.

Se encontró que $88.3 \%$ (53) de madres asistieron a 5 controles prenatales o más y $11.7 \%$ (7) no se controló el embarazo. En cuanto al control posterior al parto, $66.6 \%$ (40) de las madres asistió a un control puerperal y 33.4\% (20) no lo hizo. Se encontró que $81.7 \%$ (49) de los niños no presentaron complicaciones en los primeros días de vida y $18.3 \%$ (11) si presentó complicaciones (Bajo peso al nacer, prematurez y problemas respiratorios). 
Las edades de los niños, estaban comprendidas entre: 0 a 6 meses y 29 días un $40 \%$ (24), 7 a 12 meses y 29 días $38.4 \%$ (23), 13 a 18 meses y 29 días 10\% (6) y de 19 a 24 meses $11 \%$.(7). Se encontró que el 55\% (33) eran de sexo masculino y $45 \%$ (27) de sexo femenino. Los cuidados de los menores, son realizados en el 83.4\% (50) de los casos, por sus madres. (Ver Gráfica No. 1).

Gráfica No. 1: Persona que ofrece los cuidados al menor.

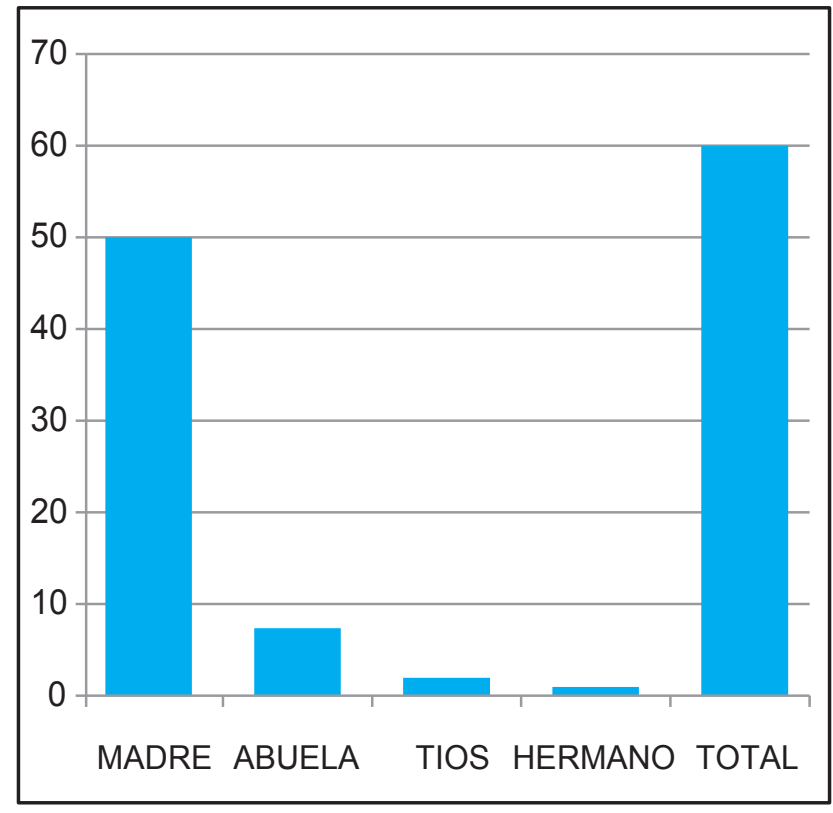

Fuente: Encuesta sobre el cuidado en los primeros mil días de vida

En relación a las enfermedades que presentaron los niños, en los últimos seis meses, se encontró que 54\% (32) habían sufrido infecciones respiratorias agudas. (Ver Tabla No. 1).

Tabla No. 1: Enfermedades de los niños, en los últimos seis meses.

\begin{tabular}{|lcc|}
\hline Enfermedades & Número $\left(\mathbf{N}^{\circ}\right)$ & Porcentaje $(\%)$ \\
\hline Respiratorias & 32 & $54 \%$ \\
\hline Gastrointestinales & 13 & $22 \%$ \\
\hline Parasitismo & 2 & $3 \%$ \\
\hline Ninguna & 8 & $13 \%$ \\
\hline Otras & 5 & $8 \%$ \\
\hline
\end{tabular}

Fuente: Encuesta sobre el cuidado en los primeros mil días de vida
El $100 \%$ de los cuidadores refirieron ofrecer cuidados higiénicos al niño, no obstante se observaron que los niños tenían déficit de higiene. El 95\% (57) de las madres/encargadas realizaron higiene en el ombligo del recién nacido y $5 \%$ (3) no. El $100 \%$ de los menores de dos años de edad fueron llevados puntualmente a vacunación, lo cual se comprobó revisando la tarjeta de vacunas.

El 93.4\% (56) de los menores de dos años recibieron lactancia materna mixta desde los primeros días y un $6.6 \%$ (4) no. Un $56.7 \%$ (34) integraron los alimentos antes de los 6 meses, $36.7 \%$ (22) entre los 6 y 12 meses y $6.6 \%$ (4) entre 1 y 2 años de edad. De las madres o encargados de los menores, 55 $(91.7 \%)$ habían realizado una o más actividades de estimulación intrauterina al feto (Ver Gráfica No. 2), 5 (8.3\%) no realizó ninguna estimulación.

Gráfica No. 2: Actividades de estimulación intrauterina.

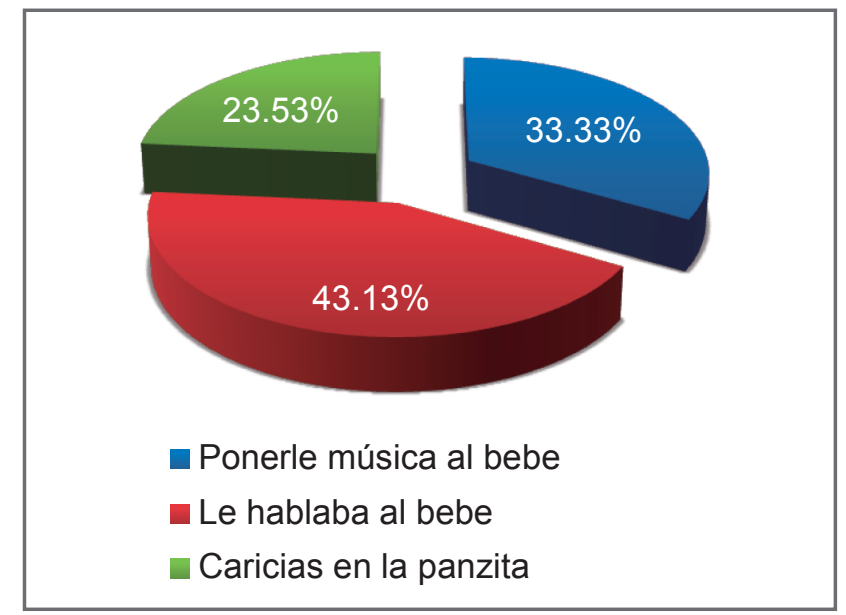

Fuente: Encuesta sobre el cuidado en los primeros mil días de vida

\section{DISCUSION}

La educación que tenga la madre o encargado influyen en los cuidados de los niños, de tal manera que se puede ver reflejado en las acciones que esta realice. Este estudio refleja que el $80 \%$ de los cuidadores de los niños tienen un nivel educativo inferior a la primaria completa en comparación con las tasas de alfabetización en Chile que son del $61 \%$ y la población adulta tiene un logro educacional 
igual o superior a la enseñanza media completa con un $96 \%$, siendo el mejor clasificado en América Latina. ${ }^{(12)}$

En Argentina, más de 99\% de las embarazadas asiste su parto en una Institución de salud y son atendidas por personal capacitado. Sin embargo, más del $10 \%$ de ellas llegan al parto sin haber tenido contacto alguno con el sistema de salud, esto es, sin control prenatal alguno. De las que se controlan, apenas una cuarta parte $(24,3 \%)$ inicia los controles precozmente durante el primer trimestre y menor aún es el número de mujeres en edad fértil que se interesaron en la preparación para emprender el embarazo. ${ }^{(13)}$ datos que se asemejan con nuestro estudio en donde $11.7 \%$ no tiene ningún control prenatal.

Las principales enfermedades que padecieron en los últimos seis meses, los niños de este estudio, fueron: infecciones respiratorias agudas (IRA) $53 \%$ (32) y las diarreas $21.6 \%$ (13) lo que se compara con las principales causas de enfermedad infantil en otros paises que son las neumonía 19\% seguido por las diarrea $17 \% .^{(14)}$ Las enfermedades diarreicas son la segunda mayor causa de muerte de niños, matando alrededor de 760, 000 niños menores de cinco años cada año y son enfermedades prevenibles y tratables. Aproximadamente 9 millones de niños menores de 5 años mueren cada año, lo que significa que muere un niño en el mundo cada 4 segundos, sin embargo, a pesar de esta alarmante cifra, la situación no está empeorando sino mejorando entre 2000 y 2010 se registró una disminución de la mortalidad infantil en un 35\%. ${ }^{(14)}$

Según los datos obtenidos, $100 \%$ de las madres o encargados refieren que realizaron cuidados higiénicos en los niños, no obstante se observaron que los niños tenían déficit de higiene. Todos los niños necesitan vivir en un entorno saludable, seguro y protector que les permita crecer y desarrollarse con normalidad y que garantice su bienestar. Los niños son especialmente vulnerables a la contaminación del aire, las sustancias químicas peligrosas, el cambio climático y las deficiencias en el suministro de agua de calidad, el saneamiento y la higiene. ${ }^{(15)}$

En la asistencia a las vacunas, la totalidad $(100 \%)$ de niños/as están vacunados de acuerdo al esquema/edad, mismo que se constató por medio de la tarjeta de vacunación. En contraste con casi dos tercios de niños que no han recibido las vacunas básicas en países que están en conflictos como en Sudan del Sur que tiene el porcentaje más alto de niños no inmunizados con $61 \%$, seguido por Somalia (58\%) y Siria (57\%). ${ }^{(16)}$

El 93.4\% (56) de los menores de dos años recibieron lactancia materna/mixta desde los primeros días de nacidos, una práctica común en nuestra población a pesar del bajo nivel económico, datos que difieren en países como Argentina, en donde el 95\% de los recién nacidos reciben leche materna exclusiva, el $54 \%$ de los bebés menores de 6 meses de edad reciben Lactancia Materna Exclusiva (LME) entre niños y niñas de 12 a 15 meses, el 61\% recibe Lactancia Materna Continuada (LMC); este número baja al $29 \%$ al llegar a los 24 meses. Se estima que la duración de LME es en promedio menor a 2 meses. La LME es mayor entre mujeres con menor educación que en comparación con el elevado nivel de riqueza (posición socio-económica). ${ }^{17)}$ Según la OMS ningún país del mundo cumple plenamente las normas recomendadas para la lactancia materna ya que según la Tarjeta de puntuación Mundial para la Lactancia materna, que evaluó las prácticas de lactancia materna en 194 naciones, encontró que solo el $40 \%$ de los menores de 6 meses reciben LME y que solo 23 países (entre ellos Perú y Bolivia) registran índices exclusivos de LME por encima del $60 \%$. $^{(18)}$

Un $56.6 \%$ de las madres/encargados de los menores integraron los alimentos antes de los 6 meses, la OMS recomienda que los lactantes empiecen a recibir alimentos complementarios a los 6 meses, primero unas dos o tres veces al día entre los 6 y 8 meses, y después, entre los 9 a 11 meses y los 12 a 24 meses, unas tres o cuatro veces al día, añadiéndoles aperitivos nutritivos una o dos veces al día, según se desee.(19) 
El 91\% (55) realizó actividades de estimulación durante el embarazo siendo un dato positivo por los beneficios ya que la intención de la estimulación es ayudar a formar un niño capaz de afrontar el futuro. Para ello es fundamental el rol que día a día desempeñan los padres, estableciendo un vínculo sólido y duradero, en un ambiente agradable y tranquilo, donde pueda moverse y sentirse feliz. ${ }^{(9)}$ De este $91 \%$ (55); $31.7 \%$ le pusieron música, $41.1 \%$ (44) le hablaban, $22.1 \%$ (24) acariciaron su vientre. Actividades similares realizaron un grupo de mujeres de Colombia: le hablaron a su bebe y realizaron estimulación auditiva un $63.8 \%$. $^{(9)}$

Conclusión Los resultados señalan que la madre o cuidadora ofrece los cuidados en los primeros mil días del niño pero estos no son totalmente adecuados y esenciales en los menores, destacan las visitas periódicas del control prenatal, estimulación durante el embarazo, asistencia en su totalidad de ni- ños en el cumplimiento de esquema de vacunación. La lactancia materna mixta, e incorporación de alimentos a temprana edad es un dato negativo que se destaca en las actividades de las madres/encargados que realizan a los menores e influyen de forma negativa en la incidencia de enfermedades gastrointestinales.

Se recomienda al personal de salud del CESAMO de Armenta y estudiantes de Enfermería de la UNAH-VS que continúen con el programa de atención integral de la niñez en la comunidad (AIN-C), realizando campañas educativas enfocadas en la implementación de los alimentos después de los seis meses, cuidados higiénicos en la preparación de los alimentos, promoción del control prenatal, promover la lactancia materna exclusiva y parto seguro para disminuir enfermedades gastrointestinales y complicaciones en los primeros mil días de vida en los niños.

\section{REFERENCIAS BIBLIOGRÁFICAS}

1. Ministerio de Salud, Colombia. (MINSALUD). Plan de acción de salud primeros 1000 días de vida Colombia (20122021). [Diapositiva Internet] Colombia: MINSALUD;2015 [citado 18 marzo 2016] 46 diapositivas. Disponible en: https:// prezi.com/pzwpdsylav_r/plan-de-accion -en-salud-primeros-1000-dias-de-vida/.

2. Organización Mundial de la Salud. OMS. Alimentación del lactante y del niño pequeño [Internet] Washington: OMS; 2016 [actualizada el 2017, citado 18 de marzo 2017]. Disponible de http://www. who.int/ mediacentre/factsheets/fs342 /es./

3. Revistazo. Veintinueve de cada 1000 niños en honduras corren el riesgo de morir antes de los 5 años. [Internet]. Tegucigalpa: Revistazo; 2013. [Actualizada 2017, citado 18 de Marzo]. disponible: http://www.revistazo.biz/web2/index. $\mathrm{php} /$ nacional/item/752-veintinueve-de -cada-1000-ni\%C3\%B1os-en-honduras corren-el-riesgo-de-morir-antes -de-los-5 -a\%C3\%B1os.
4. Ortéz, S. Atención integral en la primera infancia: una estrategia desde ChildFund América. En: Foro Mundial de Grupos de Trabajo por la Primera Infancia: Sociedad Civil - Estado. Cali: Ministerio de educación Nacional. 2009; P.46-50.

5. Casini S, Lucero Sainz GA, Hertz M, Andina E. Guía de control prenatal, embarazo normal. Rev. Hosp. Mat. Inf. Ramón Sardá. [Internet] 2002 [citado 18 de marzo]; 21(2): 51-62 Disponible en: http://www.hsaipr.com/documentos/cuidado\%20prenatal.pdf.

6. García García L M, Charrasquiel Ortiz M, Flórez Monterroza Y L, Palencia Pérez L M, Santodomingo F J, Serpa Rivera Y J, Prácticas sobre estimulación prenatal que realizan las gestantes adultas asistentes al control prenatal en Sincelejo (Colombia). Salud Uninorte 2008; 24 (1)31-39. (Fecha de consulta: 27 de septiembre de 2017). Disponible en: http:// www.redalyc.org/articulo.oa? $i d=81724$ 105. 
7. Torras, E. Lactancia materna, contacto y apego. ALBA lactancia materna.[Internet] 2015 http://albalactanciamaterna. org/lactancia/tema-6-lactancia-y-crian za/lactancia-materna-contacto-y-apego/.

8. Salcedo Abizanda S, Ribes Bautista C, Moraga Llop SA. Recién nacido cuidados de la piel. España: Asociación Española de Pediatría; [citado 18 Marzo]. Disponible en: https://www.aeped.es/ sites/default/files/documentos/recien nacido.pdf.

9. Organización Mundial de la Salud OMS. Nutrición. [Internet] Ginebra:OMS. 2011. [Actualizada el 2017, citado 18 Marzo del 2017]. Disponible en: http://www.who.int/ nutrition/topics/infantfeeding/es/

10. Secretaria de Salud, Honduras. Normas y procedimientos del programa ampliado de inmunizaciones (PAI) Honduras. [Internet] Tegucigalpa: SESAL; 2011. (Citado el 20 de Noviembre del 2016) Disponible en http://www.bvs.hn/Hondu ras/salud/normas.y.procedimientos .del.programa.ampliado.de.inmunizacio nes.\%28pai\%29.de.honduras.pdf.

11. Castillo Durán $C$, Balboa $C P$, Torrejon $S$ C, Bascuñan G C, Uauy D R. Alimentación normal del menor de 2 años. Rev. Chil Pediatr. 2013; 84(5): 565-572 (Citado el 20 de agosto del 2016). Disponible en http://www.scielo.cl/pdf/rcp/v84n5/art 13.pdf.

12. Ministerio de Educación Chile. Análisis de indicadores educativos de Chile y la OCDE en el contexto de la reforma educacional. [Internet] Chile: Ministerio de Educación; 2015 [Citado 16 septiembre 2016]. Disponible en: https://centroestu dios.mineduc.cl/wpcontent/uploads/ sites/100/2017/06/Evidencias-final_no viembre_2015.pdf.

13. Ministerio de Salud. Argentina. Recomendaciones para la Práctica del control preconcepcional, prenatal y puerperal. Argentina: Ministerio de Salud. 2013. (Julio de 2013). [citado 16 septiembre del 2016]. Disponible en: http://www. msal. gob.ar/images/stories/bes/graficos/000 0000158cnt-g02.control-prenatal. pdf.

14. Humanium. La mortalidad infantil en el mundo.[Internet] Suiza: Humanium. 2010. [Áctualizada 2017, citado 16 septiembre 2017] Disponible de: http://www. humanium.org/es/mortalidad-infantil/. Organización Mundial de la Salud. 10 datos sobre los niños y la higiene del entorno. [Internet] Ginebra: OMS; 2017 [Actualizada 2017, citado el 12 de mayo 2017] Disponible en: http://www.who.in t/features/factfiles/children_environmen tal_health/es/.

15. UNICEF. Lactancia materna y su importancia en la iniciativa Maternidades Seguras y Centradas en la familia. [Internet]. Argentina: UNICEF; 2011. [citado 16 septiembre 2016]. Disponible en: http:// www.unicef.org/argentina/spanish/Infor me_Argentina.pdf.

16. UNICEF. Dos tercios de los niños no vacunados viven en países afectados por conflictos. [Internet]. España:UNICEF;2016 (Actualizada el 2016, citado el 22 de dic. 2016) Disponible en https:// www.unicef.org/lac/media_33070.html.

17. Organización Mundial Salud. Los bebes y las madres del mundo sufren los efectos de la falta de inversión en la lactancia materna. [Internet] Ginebra: OMS;2017. (Actualizada 2017, citado el 20 de enero del 2017). Disponible en http://www.who. int/mediacentre/news/releases/2017/lack -investment-breastfeeding/es/.

18. Organización Mundial de la Salud. Alimentación Completaria. [Internet] Ginebra: OMS,2017. [Actualizada 2017, acceso el 15 de mayo del 2017] disponible en: http://www.who.int/nutrition/topi cs/complementary_feeding/es/.

19. Espinoza Ochoa R, Luna Cortes XV. Estimulación prenatal para favorecer el desarrollo del bebe en el útero. [Internet] [Tesis] Michoacan: Secretaria de Educación en el Estado. Universidad Pedagógica Nacional;2011. Disponible en http:// 200.23.113.51/pdf/29753.pdf. 